\title{
Teoría administrativa y fuerza militar: Un análisis de los principales aportes de las teorías clásicas de la administración a la gestión en las fuerzas militares
}

\author{
Carlos Ernesto Ortiz Bethés
}

\section{Resumen}

La práctica de la administración de la primera mitad el siglo XX junto con su teorización se ha visto enmarcada a la luz de tres paradigmas administrativos: El paradigma tecno-ingenieril, el paradigma sociológico y el paradigma humanista psicológico que han dado a luz las teorías clásicas de la Administración como son La Administración Científica de Taylor, La Administración Clásica de Fayol, La Teoría de las Relaciones Humanas de Mayo y La Burocracia de Weber, todas con un marco conceptual distintivo que han apuntado a la solución de los problemas organizacionales de las empresas en sus respectivos contextos históricos y que aún siguen siendo de gran utilidad, no solamente a las empresas privadas, sino también a las organizaciones estatales. De ahí que surge el análisis de este documento que presenta las principales aportaciones de las teorías clásicas de la administración de la primera mitad del siglo XX a la gestión de las fuerzas militares entorno a tres tópicos: Las tareas, las interacciones sociales y las relaciones de poder en las estructuras de orden jerárquico.

\section{Palabras Claves}

Teoría, administración, Fayol, principios administrativos, funciones administrativas, Taylor, Mayo, Weber, relaciones humanas, burocracia, fuerzas militares.

\begin{abstract}
The practice of the administration of the first half of the 20th century along with its theorization has been framed in the light of three administrative paradigms: The techno-engineering paradigm, the sociological paradigm and the humanistic psychological paradigm that have given birth to classical theories of the Administration, such as the Scientific Administration of Taylor, the Classical Administration of Fayol, The Theory of Human Relations of May and The Bureaucracy of Weber, all with a distinctive conceptual framework that have aimed at solving the organizational problems of companies in their respective historical contexts and that still continue to be very useful, not only to private companies, but also to state organizations. Hence the analysis of this document that presents the main contributions of the classical theories of the administration of the first half of the twentieth century to the management of military forces around three topics: Tasks, social interactions and relations between power in hierarchical structures.
\end{abstract}

\section{Keywords}

Theory, administration, Fayol, administrative principles, administrative functions, Taylor, Mayo, Weber, human relations, bureaucracy, military forces. 
Teoría administrativa y fuerza militar: Un análisis de los principales aportes de las teorías clásicas de la administración a la gestión en las fuerzas militares

\section{Introducción}

El presente trabajo tiene la pretensión de realizar un análisis de las principales teorías administrativas formuladas en la primera mitad del siglo XX, de su influencia y aplicabilidad en las gestiones del que hacer administrativo de las fuerzas militares colombianas. El análisis consistirá identificar los orígenes y el contexto en el que se desarrollaron las teorías de La Administración Científica de Taylor, La Administración Clásica de Fayol, La Teoría de las Relaciones Humanas de Mayo y La Burocracia de Weber; una explicación de cada teoría, una identificación de sus principales críticas, la mayoría de las escuelas de pensamiento utilizadas para la educación en administración en Colombia provienen de las teorías administrativas desarrolladas en Estados Unidos López (2012), por esto se hace necesario estudiar los postulados teóricos de los "clásicos de la administración" para poder proyectar los posibles campos de acción al interior del esquema administrativo militar. Al finalizar el documento se presentan unas proyecciones y consideraciones finales a manera de conclusión que permitirán dilucidar el aporte de estas teorías a las prácticas administrativas en el qué hacer de las fuerzas militares colombianas con miras al mejoramiento de sus procesos.

\section{La Administración en el Taylorismo}

La Administración científica o "taylorista" vino a conocerse en 1911 gracias a su principal exponente Frederick Taylor, dicha teoría partiría de un enfoque tecno-ingenieril del cual las organizaciones obtendrían como principal beneficio la optimización de recursos en las tareas y por lo tanto la eficiencia en sus operaciones Sepúlveda (2015). La Administración Científica es el primer intento de plantear una teoría acerca de la administración que hasta ese entonces era vista más cómo una técnica, pero gracias a la organización racional del trabajo se le pudo otorgar un carácter científico. Está teoría buscó dar respuesta a los problemas de productividad y eficiencia organizacional López y Arias (2006). Esta teoría tiene sus orígenes según Chiavenato (1994) en dos consecuencias de la Revolución industrial que fueron el crecimiento acelerado Ediciones EFIM y desorganizado de las empresas, que requería fuertemente la reducción de la inestabilidad y la improvisación de las operaciones, y la necesidad de aumentar la eficiencia y la competencia de las organizaciones, que buscaban maximizar sus rendimientos a la mayor expresión.

De acuerdo a Baigorri (2004), la Administración Científica de Taylor tiene tres supuestos como punto de partida: El primero es que la pérdida proviene de la ineficiencia de la mayoría de los actos diarios, el segundo es que el remedio de esa ineficiencia está en la administración sistemática y no en el personal excepcional y el tercero es que los principios de la mejor administración son aplicables a todo tipo de actividad humana y por lo tanto constituyen a la administración como una verdadera ciencia. A partir de estos supuestos se empieza a construir la estructura del Taylorismo que según Rocha, Molina, \& Ramírez (2010) se caracteriza por: “estandarización de los procesos, (...), normatización del uso de los instrumentos y de los equipos, (...) en la tarea de aumentar la eficiencia de la empresa; deja como principal responsable al jefe y relega a los trabajadores sólo al trabajo operativo".

En síntesis, la Administración Científica se resume en cuatro principios resumidos por Robbins \& Coulter (2005):

1. Establecer la ciencia de cada parte del trabajo del individuo, que reemplace el antiguo método de "hacerlo al ojo".

2. Escoger científicamente al trabajador y luego capacitarlo y desarrollarlo.

3. Cooperar entusiastamente con los trabajadores para asegurarse de que todo el trabajo se realice de acuerdo con los principios de la ciencia que se desarrolló.

4. Dividir el trabajo y la responsabilidad casi por igual entre la gerencia y los trabajadores. La gerencia asume todo el trabajo para el que está más capacitada que los trabajadores.

Así mismo, la principal herencia de ésta teoría es la organización racional del trabajo que de acuerdo a Chiavenato (1994) se basa en nueve aspectos a saber: 
1. El análisis del trabajo y estudio de tiempos y movimientos.

2. El estudio de la fatiga humana.

3. La división del trabajo y especialización del operario.

4. EL diseño de cargos y tareas.

5. La aplicación de incentivos salariales y premios por producción.

6. El concepto del homo economicus (El hombre es un ser económico que trabaja siempre a cambio de una remuneración).

7. El buen uso de las condiciones ambientales de trabajo (Entre las más destacadas esta la iluminación que garantiza mayor productividad).

8. La estandarización de métodos y de máquinas.

9. La supervisión funcional.

Una de las principales falencias del Taylorismo la identifica Sánchez (1966) cuando dice: “el taylorismo polarizó sus preocupaciones en el hombre como unidad mecánica, la concepción fisiológica de estos centros de investigación se volcó sobre el hombre como unidad biológica y descuidó los aspectos sociológicos de su comportamiento en la Empresa", en otras palabras, la visión de Taylor con respecto al obrero operaba en un reduccionismo que no le permitió analizar el potencial humano cuando este se relaciona en grupo y cómo este puede aumentar su productividad o bajarla de acuerdo a la influencia de su entorno social.

Por otra parte, el estudio taylorista de la división del trabajo también redujo la responsabilidad del obrero únicamente a las tareas dándole fuerza a la gerencia en la labor de la planeación del trabajo que, si bien da menos presión al obrero, le resta espacio para tomar iniciativa en los procesos de innovación que en la actualidad son tan necesarios.

\section{La Administración clásica de Fayol}

La teoría Clásica de la Administración data del año 1916 con Henry Fayol, según Sepúlveda (2015), Fayol identificó que la administración era una doctrina que no se había desarrollado lo suficiente en su época, de ahí que él decidió de- sarrollarla dando un gran aporte a la administración moderna, el cual consistió en dar un énfasis a la estructura administrativa y a sus funciones al interior de la empresa. En ese orden de ideas la obra de Fayol puede resumirse en tres puntos básicos. Las funciones de la empresa, el concepto de administración a partir de las funciones administrativas y los principios administrativos que vienen siendo esa estructura normativa aplicable a cualquier organización.

En cuanto a las funciones de la empresa, Fayol propone que toda empresa, sin tener en cuenta el tipo de negocio al que se dedique, cumple seis funciones esenciales:

1. Funciones técnicas: Están vinculadas a la producción de bienes y/o servicios.

2. Funciones comerciales: Están relacionadas con el intercambio, es decir, compras, ventas, permutas.

3. Funciones financieras: Consisten en el manejo del capital, la obtención de recursos financieros, pago de salarios, deudas, entre otras.

4. Funciones de seguridad: Están ligadas a la protección de los bienes de la empresa y de las personas.

5. Funciones contables: Están relacionadas con los inventarios, estados financieros, costos y estadísticas de la empresa.

6. Funciones administrativas: Están vinculadas con la integración y sincronización de las demás funciones de la empresa, de estas funciones se deriva lo que hoy se conoce como el proceso administrativo. (Chiavenato, 1994; Sepúlveda, 2015)

En lo relacionado con las funciones administrativas, Fayol propone que el proceso administrativo consiste en cinco funciones a saber:

1. Planeación: Originalmente conocido como previsión, lo cual implica anticiparse al porvenir y a partir de ahí preparar las acciones necesarias para prepararlo.

2. Organización: Consiste en darle estructura a la empresa dotándola de los recursos, sean materiales o humanos, necesarios para su funcionamiento.

Anfibios ISSN: 2665-1513 
Teoría administrativa y fuerza militar: Un análisis de los principales aportes de las teorías clásicas de la administración a la gestión en las fuerzas militares

3. Dirección: También conocido como mando, se trata de dar funcionamiento a las operaciones de la empresa por medio de la orientación al personal de la empresa.

4. Coordinación: En esta función se propone armonizar todos los esfuerzos, por lo que se hacen necesarias las reuniones semanales de jefes.

5. Control: Consiste en la verificación de que las acciones realizadas por los empleados y los procesos llevados por la empresa sean acordes con los planes trazados. (Baigorri, 2004; Chiavenato, 1994).

En lo tocante a los principios de administrativos, Fayol enuncia catorce principios que, según Robbins \& Coulter (2005) "son reglas administrativas básicas que pueden enseñarse en las escuelas y aplicarse a todas las situaciones de las organizaciones" los cuales rezan de la siguiente manera:.

1. División del trabajo: Las tareas y las personas deben ser asignadas de acuerdo al grado de especialidad con el fin de aumentar la eficiencia.

2. Autoridad: Es la facultad de tiene el gerente de dar órdenes y esperar su respectivo cumplimiento.

3. Disciplina: Todo empleado debe rendir obediencia, respeto, dedicación y energía por las tareas y las normas establecidas por la empresa.

4. Unidad de mando: Es necesario que el empleado debe estar subordinado a un solo jefe.

5. Unidad de dirección: Cada empresa y equipo de trabajo debe trabajar bajo un plan único de acción que los direccione.

6. Subordinación de los intereses de los individuos al interés general de la organización: Todos y cada uno de los intereses de los individuos que componen la empresa deben estar en función de los intereses de la empresa.

7. Remuneración: Debe existir un pago justo a empleado en relación a sus labores y responsabilidades asignadas.

8. Centralización: La facultad de tomar de decisiones reside en los altos directivos de la organización.

Ediciones EFIM
9. Cadena de escalafón: La línea de autoridad va en sentido descendente, desde el nivel más alto al más bajo.

10. Orden: Debe existir un lugar para cada cosa y cada cosa en su lugar.

11. Equidad: La gerencia debe ser justa y cortes con los empleados.

12. Estabilidad de la antigüedad personal: No es conveniente para las empresas que su personal rote demasiado, por lo cual las empresas deben buscar todos los medios posibles para garantizar la permanencia de sus empleados.

13. Iniciativa: Cuándo un empleado goza de la capacidad de idear y ejecutar planes, su compromiso y esfuerzo es mayor.

14. Espíritu de grupo: Una de las fortalezas de la empresa radica en la promoción de la armonía y la unidad del equipo de trabajo.

En cuanto a las falencias de la teoría de Fayol, se puede apreciar que en su esquema administrativo se destaca la rigidez formal, es decir, el trabajador es una variable más de la estructura administrativa por lo que la visión fayoliana es muy poco humana, así mismo la estructura piramidal de Fayol resta importancia a los sistemas de comunicación los cuales no son contemplados dentro de sus principios de administración debido a su sistema jerárquico autoritario (Baigorri 2004; Sánchez Lopéz, 1966).

\section{Las Relaciones humanas de Mayo}

Las primeras investigaciones sobre las relaciones humanas en la empresa fueron desarrolladas por Elton Mayo durante la época comprendida entre 1927 y 1932. La teoría de las relaciones humanas fue una reacción a los postulados clásicos sobre el potencial humano del cual se tenía la noción de taylorista y fayolista que consideraba al comportamiento humano en la organización como una variable explicada por motivaciones basadas en los incentivos económicos Rocha et al. (2010).

El objetivo de las investigaciones de Mayo era identificar como el empleado podía lograr el ser más productivo, teniendo en cuenta que los postulados clásicos solo podían explicar la pro- 
ductividad a través del pago de remuneraciones altas, Mayo cambia el concepto del hombre económico de Taylor y propone el concepto del hombre social que encuentra una explicación a su comportamiento a partir de la motivación y la satisfacción personal Rocha et al.(2010).

Las investigaciones de Mayo se dieron en el contexto de los esfuerzos de la Western Electric Company, en colaboración con el National Research Council, donde había intentado conocer los efectos del contorno físico sobre la productividad de los trabajadores los cuales no reflejaron un alto grado de impacto sobre esta. Dicho fracaso provocó que el estudio de la influencia del contorno social sobre el desempeño de los individuos Sánchez (1966), dadas estas investigaciones se dio origen a un nuevo enfoque llamado popularmente humanístico pero también visto como un paradigma psicológico tecno-instrumental según Sepúlveda (2015).

Las investigaciones de la Western Electric y Mayo se llevaron en la fábrica de Hawthorne, razón por la que son más conocidas por el nombre de los Estudios de Hawthorne. Dicho estudio se llevó a cabo en cuatro fases a saber según lo explica Chiavenato (1994):

1. Efectos de iluminación: En esta fase el fin era el conocer el efecto de los cambios de luz en las condiciones de trabajo sobre la productividad del empleado. El resultado obtenido fue que el ser humano reacciona de una manera más compleja que las máquinas, por lo que fracasó la noción mecanicista del ser humano proveniente del Taylorismo.

2. Sala de armado de relés: El fin de esta fase era el conocer el efecto de los cambios en las condiciones de trabajo en la búsqueda de explicar la fatiga y la monotonía. Se pudo identificar que cuándo el supervisor de los grupos se encuentra integrado con los demás trabajadores y el ambiente amistoso sin presiones impera, la estabilidad del equipo aumenta trayendo como consecuencia la disminución de la fatiga, la monotonía y las faltas demostrándose la influencia del grupo sobre el rendimiento individual.

3. Programa de entrevistas: El propósito de 16 la tercera fase era conocer más a los empleados, escuchar sus opiniones y sugerencias, el resultado fue la identificación de la presencia del poder informal del en los empleados, el comportamiento del trabajador a nivel individual es distinto cuando se encuentra en un rol grupal.

4. Montaje de terminales: La última fase de le experimento buscaba analizar la organización informal en los empleados, dándose cuenta que el poder de esta era de tal magnitud en el rendimiento de los empleados hasta el punto de observar que el ritmo de trabajo estaba en función de lo que el grupo decidía.

Lastimosamente el experimento de Hawthorne vio el cese de actividades debido a problemas financieros, sin embargo la información obtenida en las cuatro fases fueron las siguientes Chiavenato (1994):

1. El nivel de producción está determinado por normas sociales y las expectativas que lo rodean.

2. El comportamiento del individuo de apoya en el grupo.

3. Los estándares sociales controlan el comportamiento del trabajador.

4. El poder de la organización social-informal es más fuerte que la formal.

5. En las organizaciones es importante tener en cuenta los aspectos y decisiones que tienen los empleados para crear relaciones humanas.

6. A mayor interacción, mayor capacidad productiva.

7. Cualquier cambio en la empresa produce una reacción dentro del personal.

8. Mientras mejor se siente el empleado en su puesto de trabajo es más productivo.

Según Sánchez (1966), el aporte de esta teoría a la administración en general es el ver la empresa como un sistema de interacción social en el que se necesita que los objetivos de la empresa y de la dirección en general deben estar en perfecto equilibrio con los objetivos de la organización informal de la empresa para que haya estabilidad en el desempeño organizacional.

Anfibios ISSN: 2665-1513 
Teoría administrativa y fuerza militar: Un análisis de los principales aportes de las teorías clásicas de la administración a la gestión en las fuerzas militares

Baigorri (2004) plantea que las principales críticas a las Relaciones Humanas de Mayo son las siguientes: En primera medida, Mayo tiene en su teoría la idea implícita de considerar a la empresa como un sistema cerrado puesto que no se tienen en cuenta las relaciones de la empresa con las fuerzas económicas, sociales, políticas y culturales del entorno en el que se desempeña, reduciéndola solamente a las interacciones sociales en su interior. Por otro lado, el propósito de generar satisfacción en el empleado, plantea Baigorri, es el de aplacar los movimientos sindicales que tanto problema generan a las empresas modernas, por lo que se hace necesario buscar un mecanismo que mantenga a la clase obrera contenta y los empresarios puedan seguir aprovechando el trabajo de la mano de obra.

\section{La Burocracia de Webber}

En 1947 se volvió popular entre los medios académicos de la administración la teoría burocrática de Weber, a pesar de haber sido planteada desde varios años atrás no había gozado de popularidad debido al auge de las teorías anteriormente expuestas.

$\mathrm{Su}$ origen debe a las siguientes razones expuestas por Chiavenato (1994):

1. La falta de acuerdo de las teorías clásicas de la administración y las relaciones humanas que carecían de un elemento integrador para ver el contexto completo de la empresa en sus dimensiones laborales y sociales.

2. La necesidad de un modelo que fuera capaz de involucrar las tareas y las relaciones sociales en la empresa.

4. El constante crecimiento y la complejidad de las organizaciones.

5. El resurgimiento de la sociología de la burocracia.

Según Robbins \& Coulter (2005) es “una forma de organización caracterizada por la división del trabajo, una jerarquía bien definida, reglas y normas detalladas, y relaciones impersonales". A su vez las características distintivas de este sistema que identifica Baigorri (2004) son las siguientes:

Ediciones EFIM
1. Una clara jerarquía de autoridad donde se identifican posiciones organizadas en un esquema piramidal donde cada cargo de nivel superior tiene control y supervisión del inferior.

2. La conducta de los individuos en la burocracia está dada por la observancia de reglas y procedimientos de estricto cumplimiento que le aportan a la organización un carácter supremamente formal.

3. Los funcionaros de una estructura burocrática deben ser asalariados a tiempo completo, en cada posición jerárquica hay una retribución fija donde los puestos de trabajo están divididos en tareas simples y rutinarias.

4. La promoción o selección del personal debe ser a partir de méritos tales como la capacidad técnica, la antigüedad o ambas cosas.

5. Las tareas son de carácter impersonal, es decir, hay una clara separación entre el rol del funcionario en la organización y su vida afuera de esta.

6. Ningún miembro de la estructura burocrática es dueño o posee algo de la unidad de trabajo.

Este modelo de administración fue adoptado por la necesidad de encontrar un elemento integrador en las organizaciones que abarcara la complejidad de los diferentes elementos constitutivos de las empresas que son las tareas, las interacciones sociales y las relaciones de poder Sánchez (1966). No obstante, ya no es tan popular en las empresas privadas, esta filosofía administrativa ha migrado hacia los entes estatales que están más acostumbrados a tratar con el tema de las relaciones de poder y la normatización de reglamentos y procedimientos.

Las principales debilidades de esta teoría radican en que el exagerado apego a los reglamentos genera lentitud en los procesos empresariales, por ende a la falta de flexibilización de los procedimientos las empresas optan por acoger posturas epistemológicas que les permitan gestionar mejor su velocidad de respuesta frente a las condiciones del entorno del cual, por ser de naturaleza cambiante, se hace necesario innovar y realizar cambios rápidos para adaptarse a las necesidades que se presenten. De ahí que se presenta la noción popular de las empresas y organizaciones estatales que tienden a ser muy lentas y poco competitivas. 


\section{Perspectivas}

Aterrizando la práctica administrativa en el contexto de las fuerzas militares, existe la noción de que existen dos sistemas: En las organizaciones que no pertenecen al Estado existe la Administración privada y en el Estado la Administración pública, no obstante Jimenéz (1977) establece que no existen dos tipos de administraciones, solamente existe una Administración que a su vez tiene dos subsistemas que pertenecen al sector público y privado. De esta afirmación se puede inferir que por el hecho de que se haya teorizado acerca de la administración en los sectores privados de la economía, no se puede excluir este aporte cognoscitivo a la ciencia administrativa pública. En otras palabras, los modelos administrativos provenientes de teóricos del sector privado son una herramienta muy útil para las prácticas administrativas dentro del sector público y militar.

En cuanto a la composición de la fuerza pública colombiana, está consta del Ejercito, la Armada, la Fuerza Aérea y la Policía Nacional; estas fuerzas se han visto durante muchos años enfrentadas a fenómenos como la lucha contra el narcotráfico, las guerrillas del conflicto armado, los grupos paramilitares y poderosas organizaciones criminales que han traído grandes consecuencias en la sociedad colombiana Schultze (2012). Estas no tienen una relación con los gobernantes civiles caracterizada por un control civil firmemente institucionalizado, antes bien, según Schultze (2012), "estas relaciones son producto de una simbiosis cívico-militar que le da a fuerza pública un amplio margen de maniobra y decisión en al ámbito público del país".

Dadas estas circunstancias la fuerzas militares colombianas tienen además de una labor de protección a la sociedad civil del Estado, unas labores administrativas que cumplir en las que se ven implicadas las relaciones de poder, las aplicaciones de reglamentos, la asignación de recursos y esfuerzos para la ejecución de sus labores, la división del trabajo administrativo y militar, la identificación de los procesos clave de su organización y el rol de su actividad en la obtención de los logros esperados para la construcción y desarrollo del país. La estructu- ra organizacional de las fuerzas militares está determinada por líneas de mando dónde la autoridad es repartida de una manera verticalmente descendente, por esta razón las teorías administrativas clásicas pueden ser de gran utilidad en las gestiones de la práctica administrativa de las fuerzas militares, debido a que fueron propuestas empresas con estructuras organizacionales similares. En la siguiente tabla se puede apreciar cómo pueden aportar las teorías administrativas de la primera mitad del siglo XX a la gestión administrativa militar:

\section{Tabla 1: Cuadro de análisis de las "Teorías tradicionales de la Administración"}

\begin{tabular}{|c|c|c|c|c|}
\hline Teoría & $\begin{array}{l}\text { Adminis- } \\
\text { tración } \\
\text { Científica }\end{array}$ & $\begin{array}{l}\text { Adminis- } \\
\text { tración } \\
\text { Clásica }\end{array}$ & $\begin{array}{l}\text { Escuela } \\
\text { de las } \\
\text { Relaciones } \\
\text { Humanas }\end{array}$ & Burocracia \\
\hline Autores & $\begin{array}{l}\text { Frederick } \\
\text { Taylor }\end{array}$ & $\begin{array}{l}\text { Henry } \\
\text { Fayol }\end{array}$ & $\begin{array}{l}\text { Elton } \\
\text { Mayo }\end{array}$ & $\begin{array}{l}\text { Max } \\
\text { Weber }\end{array}$ \\
\hline Énfasis & $\begin{array}{l}\text { La eficien- } \\
\text { cia del uso } \\
\text { de recursos } \\
\text { en la ejecu- } \\
\text { ción de las } \\
\text { tareas. }\end{array}$ & $\begin{array}{l}\text { El papel } \\
\text { de las } \\
\text { funciones y } \\
\text { estructura } \\
\text { administra- } \\
\text { tiva. }\end{array}$ & $\begin{array}{l}\text { La moti- } \\
\text { vación y } \\
\text { satisfac- } \\
\text { ción en el } \\
\text { trabajo a } \\
\text { través la } \\
\text { interacción } \\
\text { social. }\end{array}$ & $\begin{array}{l}\text { La impor- } \\
\text { tancia de la } \\
\text { normatiza- } \\
\text { ción de los } \\
\text { reglamen- } \\
\text { tos y pro- } \\
\text { cedimien- } \\
\text { tos en las } \\
\text { estructuras } \\
\text { organiza- } \\
\text { cionales. }\end{array}$ \\
\hline Paradigmas & $\begin{array}{l}\text { Tecno-in- } \\
\text { genieril }\end{array}$ & $\begin{array}{l}\text { Tecno-in- } \\
\text { genieril }\end{array}$ & $\begin{array}{l}\text { Humanis- } \\
\text { mo psico- } \\
\text { lógico }\end{array}$ & $\begin{array}{l}\text { Socioló- } \\
\text { gico }\end{array}$ \\
\hline $\begin{array}{l}\text { Aportes a } \\
\text { la gestión } \\
\text { administra- } \\
\text { tiva militar }\end{array}$ & $\begin{array}{l}\text { El estudio } \\
\text { científico } \\
\text { de las } \\
\text { tareas. }\end{array}$ & $\begin{array}{l}\text { La especia- } \\
\text { lización del } \\
\text { trabajo por } \\
\text { funciones. }\end{array}$ & $\begin{array}{l}\text { El trabajo } \\
\text { con los } \\
\text { grupos } \\
\text { informales } \\
\text { dentro del } \\
\text { esquema } \\
\text { militar }\end{array}$ & $\begin{array}{l}\text { El uso } \\
\text { adecuado } \\
\text { de las rela- } \\
\text { ciones de } \\
\text { poder y el } \\
\text { estableci- } \\
\text { miento de } \\
\text { las normas } \\
\text { de trabajo. }\end{array}$ \\
\hline
\end{tabular}

Fuente: Elaboración propia

Teniendo en cuenta los aportes que las distintas teorías de la administración pueden hacer a la gestión administrativa de las fuerzas armadas, es importante adoptar una postura integradora de todas ellas, es decir, evitar el ser excluyente al momento de utilizar uno de estos enfoques ya que el uso en conjunto de estas teorías pueden dar como resultado una gestión realmente eficiente que integre los elementos constitutivos de una organización que son: Las tareas, las interacciones sociales y las relaciones de poder en las estructuras jerárquicas.

Anfibios ISSN: 2665-1513 
Teoría administrativa y fuerza militar: Un análisis de los principales aportes de las teorías clásicas de la administración a la gestión en las fuerzas militares

Uno de los errores más comunes que se comenten en las organizaciones a la hora de adoptar modelos administrativos es la falta de integración de los distinto enfoques administrativos, por ejemplo, caer en el uso excesivo del modelo administrativo taylorista o fayolista implica una marcada tendencia a medir los procesos y las tareas, en el caso de las fuerzas militares, esto llevaría a un control permanente sobre las operaciones tanto militares y administrativas y corren el riesgo de invisibilizar la parte humana del trabajo de cada uno de los integrantes de este organismo social. Olvidar el componente humanismo en el esquema administrativo es no prestarle atención a influencia del grupo sobre el individuo, es pasar por alto los comportamientos que tienen las personas en sus actividades diarias y a su vez influyen en la eficiencia de las tareas.

En ese mismo orden de ideas, si el modelo administrativo solo se centrara en las interacciones humanas y su influencia en la productividad de las personas, también tendría sus limitantes, puesto que este enfoque no da una respuesta a los problemas de eficiencia y eficacia que tienen que resolver las instituciones militares en lo relacionado con el uso de los recursos para la obtención de sus metas.

De la misma manera el enfoque burocrático posee limitantes, la excesiva normatización y reglamentación han traído como consecuencia el apego de los individuos hacia el poder. Una vez invisibilizado las interacciones sociales en este modelo, aquellos que tienen posiciones de alto poder jerárquico en el modelo administrativo militar tienden a maltratar a sus subalternos y a realizar abusos de poder escondidos en su posición de mando y jerarquía, ocasionando un aumento de la tensión en estos puestos de trabajo y por ende un crecimiento en los factores de riesgo psicosociales en los puestos de trabajo pertenecientes a las instituciones militares.

Los modelos administrativos son respuestas a diferentes problemáticas que se han presentado en diferentes contextos que han requerido de su presencia en las organizaciones. En las fuerzas militares es necesario integrar cada uno de estos Ediciones EFIM modelos para poder disponer de una visión amplia y compleja de las realidades que se viven al interior de su institucionalidad en la actualidad. Problemas como la lucha de poderes y egos, el manejo de conflictos, la optimización de los recursos y el cumplimiento de las normas no se pueden solucionar con una visión sesgada a un paradigma de la administración, es necesario hacer uso del eclecticismo para aplicar modelos administrativos integrales que sean lo suficientemente potentes para que sean funcionales dentro de las fuerzas militares.

\section{Conclusiones}

Las teorías administrativas clásicas provenientes de la primera mitad del siglo XX han sido ampliamente utilizadas en el sector privado por las empresas, no obstante esto no es una razón para excluirlas del análisis de la gestión administrativa de las organizaciones públicas. En el caso de las fuerzas militares, acoger los paradigmas de la administración clásica puede traer como beneficio a su práctica administrativa la optimización de recursos, una división del trabajo basada en el análisis científico de las capacidades de los individuos que integran el aparato militar, una eficiente gestión del personal a partir de la correcta canalización de las interacciones sociales que se presentan en los grupos informales que se van conformando en la entidad y por último, una concepción global de cómo establecer reglamentos basados en las diferentes jerarquías y relaciones de poder en el interior de las fuerzas armadas.

Es muy importante para las fuerzas militares adoptar modelos administrativos que sean capaces de resolver problemas de alta complejidad que integren realidades de tipo praxeológico, ontológico, axiológico y epistémico para la solución de sus problemas y el cumplimiento de sus metas como institución. El propósito de tener un enfoque ecléctico de las posturas clásicas de la administración radica esencialmente en integrar las variables tareas, interacciones sociales y relaciones de poder para darle eficiencia óptima al modelo administrativo de las fuerzas militares. 
Finalmente, las buenas prácticas administrativas provienen de la correcta aplicación de la teoría en el contexto actual, es decir, para que los paradigmas clásicos puedan tener incidencia en el mundo moderno de la administración en las fuerzas militares es necesario que sean contextualizados sus postulados en los diferentes niveles de mando de la estructura organizacional, empezando por los altos mandos hasta el personal del nivel más bajo en cuanto a jerarquía ya que esto es lo que le da viabilidad a los modelos administrativos, la apropiación de la epistemes administrativa reflejada en la praxis del qué hacer cotidiano.

\section{Referencias bibliográficas}

Baigorri, A. (2004). Introducción a la sociología de la empresa. (IVES/Sharebooks, Ed.).

Chiavenato, I. (1994). Introduccion a la Teoría General de la Administración. (M.-H. I. Editores, Ed.) (Séptima ed). México.

Jimenéz Nieto, J. I. (1977). Teoría Administrativa del Gobierno. (U. del Pacífico, Ed.) (Primera ed). Lima.

López, F. (2012). Educación en administración y modas administrativas en Colombia. Revista Universidad EAFIT.

Maria Esperanza López, Leonel Arias, S. R. (2006). Las organizaciones y la evolucion administrativa. Scientia et Technica Año XII, (31), 147-152.

Robbins, S., \& Coulter, M. (2005). Administración. (P. Educación, Ed.) (Octava Edi). México. Retrieved from https://www.academia.edu/11095692/Administracion_8va_Edicion_-_Stephen_P._Robbins_y_Mary_Coulter

Rocha Valencia, L. A., Molina Parra, P. A., \& Ramírez Díaz, L. F. (2010). Del dicho al hecho: Una discusión acerca del devenir histórico de las teorías administrativas y su correspondencia con las prácticas empresariales. Revista Politécnica, 11, 57-72.
Sánchez Lopéz, F. (1966). LA EMPRESA COMO UNIDAD DE ANÁLISIS, I(1).

Schultze-Kraft, M. (2012). La cuestión militar en Colombia : la fuerza pública y los retos de la construcción de paz. Construcción de Paz En Colombia, (January 2012), 405-433. https:// doi.org/10.7440/2012.36

Sepúlveda Fernández, R. de J. (2015). Discusiones Filosóficas en los Paradigmas de Formación Administrativa: Estudio comparativo en programas de administración del Eje Cafetero Colombiano. (U. T. de Pereira, Ed.) (Primera ed). Pereira. 